THE ASTROPHYSICAL JOURNAL, 564:887-895, 2002 January 10

(C) 2002. The American Astronomical Society. All rights reserved. Printed in U.S.A.

\title{
THE SPECTRAL ENERGY DISTRIBUTION OF HH 30 IRS: CONSTRAINING THE CIRCUMSTELLAR DUST SIZE DISTRIBUTION
}

\author{
Kenneth Wood, ${ }^{1,2}$ Michael J. WolfF, ${ }^{3}$ J. E. BJorkman, ${ }^{4}$ and Barbara Whitney ${ }^{3}$ \\ Received 2001 June 14 ; accepted 2001 September 10
}

\begin{abstract}
We present spectral energy distribution (SED) models for the edge-on classical T Tauri star HH 30 IRS that indicate that dust grains have grown to larger than $50 \mu \mathrm{m}$ within its circumstellar disk. The disk geometry and inclination are known from previous modeling of multiwavelength Hubble Space Telescope images, and we use the SED $(0.5 \mu \mathrm{m} \leq \lambda \leq 3 \mathrm{~mm})$ to constrain the dust size distribution. Model spectra are shown for different circumstellar dust models: a standard interstellar medium (ISM) mixture and larger grain models. As compared to ISM grains, the larger dust grain models have a shallower wavelength-dependent opacity: smaller at short wavelengths and larger at long wavelengths. Models with the larger dust grains provide a good match to the observed SED of HH 30 IRS. Although the currently available SED is poorly sampled, we estimate $L_{*} \approx 0.2 L_{\odot}, M_{\text {disk }} \approx 1.5 \times 10^{-3} M_{\odot}$, and a power law with exponential cutoff dust grain size distribution. This model provides a good fit to the currently available data, but mid- and far-IR observations are required to more tightly constrain the size distribution. The accretion luminosity in our models is $L_{\text {acc }} \lesssim 0.2 L_{*}$, corresponding to an accretion rate $\dot{M} \lesssim 4 \times 10^{-9} M_{\odot} \mathrm{yr}^{-1}$. Dust size distributions that are simple power-law extensions (i.e., no exponential cutoff) yield acceptable fits to the optical/near-IR but too much emission at millimeter wavelengths, and require larger disk masses up to $M_{\text {disk }} \sim 0.5 M_{\odot}$. Such a simple size distribution would not be expected in an environment such as the disk of HH 30 IRS (i.e., where coagulation and accretion processes are occurring in addition to grain shattering), particularly over such a large range in grain sizes. Its ability to adequately characterize the grain populations, however, may be determined from more complete observational sampling of the SED in the mid- to far-IR.
\end{abstract}

Subject headings: accretion, accretion disks - dust, extinction - radiative transfer - scattering stars: individual (HH 30 IRS) - stars: pre-main-sequence

\section{INTRODUCTION}

The Hubble Space Telescope (HST) Wide Field Planetary Camera 2 (WFPC2) images of HH 30 IRS (Burrows et al. 1996) revealed the characteristic scattered-light pattern of an edge-on disk in a classical T Tauri system: two almost parallel nebulae separated by an opaque dust lane (e.g., Whitney \& Hartmann 1992). Detailed modeling of the WFPC 2 images enabled Burrows et al. (1996) to constrain the size, shape, and optical depth of the disk. In addition, their best model images were constructed using dust grains with a scattering phase function that is more forward throwing than typical interstellar medium (ISM) grains, indicative of grain growth within the disk (Hansen \& Travis 1974).

Wood et al. (1998) presented scattered-light models of edge-on disks showing that the width of the dust lane decreases toward longer wavelengths where the dust is less opaque (assuming a wavelength dependence for absorption and scattering of typical ISM dust grains). They predicted that the HH 30 IRS nebulosity should appear almost pointlike at the $K$ band. The new Near-Infrared Camera and Multi-Object Spectrometer (NICMOS) observations of

\footnotetext{
${ }^{1}$ School of Physics and Astronomy, University of St. Andrews, North Haugh, St. Andrews, Kingdom of Fife, KY16 9AD, Scotland; kw25@standrews.ac.uk.

${ }^{2}$ Harvard-Smithsonian Center for Astrophysics, 60 Garden Street, Cambridge, MA 02138; kwood@cfa.harvard.edu.

${ }^{3}$ Space Science Institute, 3100 Marine Street, Suite A353, Boulder, CO 80303; bwhitney@colorado.edu, wolff@colorado.edu.

${ }^{4}$ Ritter Observatory, Department of Physics and Astronomy, University of Toledo, Toledo, $\mathrm{OH} 43606$; jon@astro.utoledo.edu.
}

Cotera et al. (2001), however, show a distinct dust lane even at the longest NICMOS wavelength. Cotera et al. used the NICMOS images to study the near-IR wavelength dependence of the circumstellar dust opacity. They modeled the NICMOS images using a dust size distribution that has a smaller near-IR opacity and a shallower wavelength dependence than ISM dust. Their modeling showed that the extinction through the disk to the central source is $A_{V}=$ 7900. The derivation of this extinction requires detailed radiation-transfer modeling, since the $H S T$ images are dominated by scattered light so standard reddening estimates are not applicable.

The scattered-light models of Burrows et al. (1996), Wood et al. (1998), and Cotera et al. (2001) fit the width of the dust lane, from which they derive $M_{\text {disk }} \gtrsim 10^{-4} M_{\odot}$. As noted by Burrows et al., what is really determined from scatteredlight modeling is the equatorial optical depth in the disk midplane, $\tau_{\text {eq }} \propto \kappa_{\lambda} M_{\text {disk }}$. The mass determination is therefore very uncertain since it depends on the adopted grain size distribution and opacity. Scattered-light models of the edge-on disk system HK Tauri/c derive $M_{\text {disk }} \approx 10^{-4} M_{\odot}$ assuming ISM dust (Stapelfeldt et al. 1998). D'Alessio, Calvet, \& Hartmann (2001), however, have shown that the edge-on disk of HK Tau/c may be modeled with a massive disk, $M_{\text {disk }}=0.065 M_{\odot}$, in which the maximum grain size is $1 \mathrm{~m}$. Their results also demonstrate the utility of longwavelength observations for distinguishing between a lowmass disk with ISM grains and a massive disk with a population of much larger grains.

It is difficult to draw firm conclusions on circumstellar dust properties since the often unknown inclination and disk geometry lead to degeneracies in SED models. For HH 
30 IRS, however, scattered-light modeling provides the disk geometry and inclination. Therefore, the circumstellar grain properties may be studied through detailed modeling of its SED that covers the range $0.55 \mu \mathrm{m} \leq \lambda \leq 3 \mathrm{~mm}$ (Stapelfeldt \& Moneti 1999). This paper presents model SEDs for HH 30 IRS that highlight the differences between ISM and larger dust grain models. Our modeling adopts the disk structure derived from fitting $H S T$ images and calculates the radiative equilibrium SED from a star + disk system. We use our new Monte Carlo radiative equilibrium code for the calculations and show SED models at different inclination angles ranging from almost edge-on (appropriate to HH 30 IRS) to pole-on viewing of the star + disk system.

Section 2 presents the HH 30 IRS SED taken from the literature; $\S 3$ describes the radiation transfer, disk model, and dust opacity; and $\S 4$ presents SED models for a passive reprocessing disk and also the effects of including viscous accretion luminosity. Section 5 summarizes our results.

\section{THE SPECTRAL ENERGY DISTRIBUTION OF HH 30 IRS}

Our models are constructed with the goal of reproducing the currently available SED for HH 30 IRS. The SED displayed in Figure 1 is taken from Mundt \& Fried (1983: $V$ and I); Vrba, Rydgren, \& Zak (1985: H, K, and an upper limit at $L$ ); and Reipurth et al. (1993: $1.3 \mathrm{~mm})$. The SED measured by Infrared Space Observatory (ISO) between 4.5

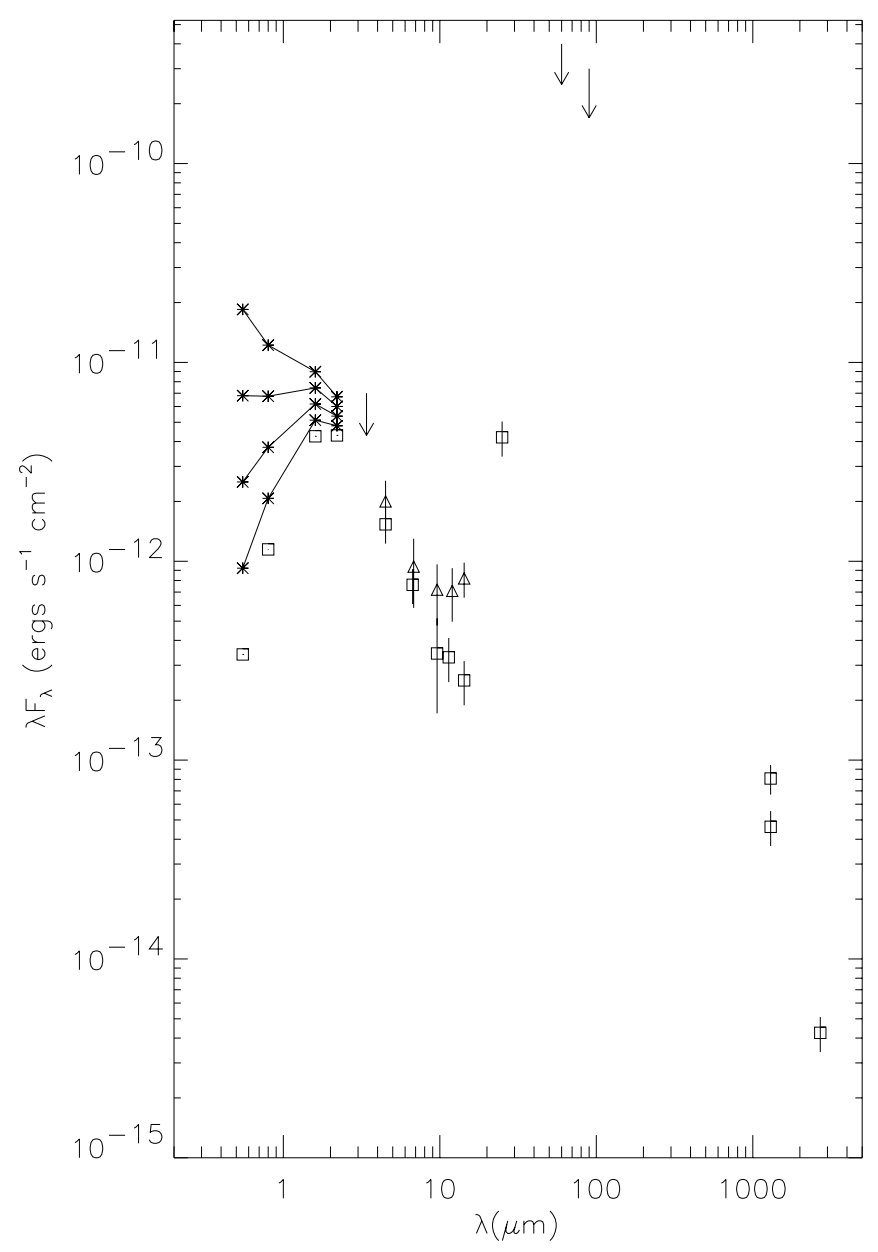

FIG. 1.-Spectral energy distribution of HH 30 IRS. Triangles are the ISO reductions of Brandner et al. (2000). The ISO fluxes at 60 and $90 \mu \mathrm{m}$ are upper limits. Also shown are dereddened SEDs (stars) assuming $1 \leq$ $A_{V} \leq 4$, with $A_{V}=1$ the lowest curve. and $90 \mu \mathrm{m}$ and additional millimeter fluxes (Stapelfeldt \& Padgett 2001) were presented by Stapelfeldt \& Moneti (1999, Fig. 3).

The ISO data were also presented by Brandner et al. (2000), and their reduction produced slightly larger fluxes than that of Stapelfeldt \& Moneti (1999). Both reductions are shown on this figure. The fluxes in Figure 1 were not taken simultaneously, and HH 30 IRS is known to vary in the optical by up to $1.4 \mathrm{mag}$ at $V$, but this amplitude decreases toward longer wavelengths (Wood et al. 2000). Consequently, this variability will not affect our dust size distribution modeling since the different dust models are discriminated at long wavelengths $(\lambda \gtrsim 20 \mu \mathrm{m})$, where any variability is expected to be small.

Although the 60 and $90 \mu \mathrm{m}$ data are upper limits, the SED shows the characteristic double-peaked spectrum of an edge-on disk: the direct starlight is obscured by the disk, the near-IR peak arises from scattered light, and the far-IR peak is the result of reprocessing of stellar radiation in the disk and possibly the liberation of disk accretion luminosity (e.g., Preibisch, Sonnhalter, \& Yorke 1995; Boss \& Yorke 1996; Men'shchikov \& Henning 1997; D'Alessio et al. 1999).

The larger flux measurement at $1.3 \mathrm{~mm}$ is from Reipurth et al. (1993) and was observed with a $23^{\prime \prime}$ beam. The millimeter data from Stapelfeldt \& Moneti (1999) are from high spatial resolution mapping of the HH 30 IRS disk using the Owens Valley Radio Observatory (OVRO). As such, the OVRO data probes emission from the disk as opposed to the unresolved data of Reipurth et al. (1993). The larger flux measurement of Reipurth et al. indicates that there is material beyond the disk observed by HST and OVRO, but the large beam size does not spatially resolve this emission or determine whether all of it is really associated with $\mathrm{HH}$ 30 IRS (see also the $1.3 \mathrm{~mm}$ map of Motte \& Andre 2001, their Fig. 1h). In our modeling, we are specifically interested in the grain properties within the resolved circumstellar disk, and therefore our models fit the millimeter data of Stapelfeldt \& Moneti (1999). The HST images are fit with $R_{\text {disk }} \sim 200 \mathrm{AU}$ (Cotera et al. 2001), which is comparable to the extent of the millimeter data we are modeling (Stapelfeldt \& Padgett 2001), and we adopt this outer radius (see §3.2). Both $1.3 \mathrm{~mm}$ points are shown in Figure 1, but in subsequent figures only the OVRO data are shown.

The foreground extinction to HH 30 IRS is not well determined as the central source is not directly visible at optical and near-IR wavelengths. Cotera et al. (2001) estimated $A_{V}<4$, and Figure 1 shows dereddened SEDs for $1 \leq A_{V} \leq 4$ using the dereddening formulae of Cardelli, Clayton, \& Mathis (1988) with $R_{V}=3.1$. Comparing the dereddened SED to models of edge-on disks suggests $A_{V}<2$.

\section{MODELS}

We now outline the radiation transfer technique, disk geometry, luminosity sources, and circumstellar dust properties that are required in our modeling.

\subsection{Radiative Equilibrium Calculation}

The radiation transfer calculations use the Monte Carlo radiative equilibrium and temperature correction technique of Bjorkman \& Wood (2001), which we have adapted to simulate T Tauri disks (J. E. Bjorkman, K. Wood, \& B. Whitney 2001, in preparation). The output of our code is the disk temperature structure (due to heating by stellar 
photons and accretion luminosity) and the emergent SED at a range of viewing angles. A calculation of the hydrostatic disk structure (e.g., D'Alessio et al. 1999) can be included in the Monte Carlo technique, but this would require an iterative scheme. At present we have not implemented such a scheme and instead perform the radiative equilibrium calculation within a fixed disk geometry as we now describe.

\subsection{Disk Structure}

We calculate model SEDs by performing the radiation transfer and radiative equilibrium calculation within the disk structure derived from scattered-light modeling of the HST images of HH 30 IRS. The HST images have been modeled by Burrows et al. (1996), Wood et al. (1998), and Cotera et al. (2001) with a flared disk density structure,

$$
\rho=\rho_{0}\left(\frac{R_{*}}{\varpi}\right)^{\alpha} \exp -\frac{1}{2}\left[\frac{z}{h(\varpi)}\right]^{2},
$$

where $\varpi$ is the radial coordinate in the disk midplane, $\rho_{0}$ is the density at the stellar surface, and the scale height increases with radius, $h=h_{0}\left(\varpi / R_{*}\right)^{\beta}$. For our SED modeling we adopt $\beta=1.25, \alpha=2.25, h_{0}=0.017 R_{*}$, giving $h(100$ $\mathrm{AU})=17$ AU. The $\beta$ - and $\alpha$-values are consistent with detailed hydrostatic structure calculations (D'Alessio et al. 1999). The inner radius, $R_{0}=6 R_{*}$, is set to be the dust destruction radius for $T_{*}=3500 \mathrm{~K}$ and dust sublimation temperature $T_{d}=1600 \mathrm{~K}$. The outer disk radius is fixed at $R_{\text {disk }}=200 \mathrm{AU}$ to match the spatial extent of the HST images and the millimeter observations of Stapelfeldt \& Padgett (2001). Therefore, our SED modeling, derived disk mass, and dust properties apply to the material within $R_{\text {disk }}=200 \mathrm{AU}$, which forms the observed SED. More dust likely exists beyond $200 \mathrm{AU}$ (Reipurth et al. 1993; Motte \& Andre 2001), but it is not detected in the high spatial resolution $H S T$ or millimeter images and therefore does not contribute to the observed SED we are modeling.

To fit the NICMOS F160W image, Cotera et al. (2001) used a slightly different geometry $(\beta$ and $\alpha$ ) from what we described above and derived $M_{\text {disk }}=6.7 \times 10^{-4} M_{\odot}$. This scattered-light modeling probes the mass of small particles that dominate the opacity at short wavelengths. As such, modeling of optical and near-IR images is insensitive to large particles, rocks, and planetesimals, and therefore this mass is a lower limit.

\subsection{Energy Sources}

The energy input to the disk is from stellar photons and accretion luminosity liberated in the disk. Initially we consider a passive disk heated by a uniformly bright star. Section 4.3 places limits on the luminosity arising from viscous accretion. As discussed in the previous section, the disk structure is fixed for our radiation transfer simulations. We then use $\alpha$-disk theory (Shakura \& Sunyaev 1973) to determine the accretion rate for a given disk mass and structure. The accretion rate and viscosity parameter, $\dot{M}$ and $\alpha_{\text {disk }}$, are related to the disk parameters by (see Bjorkman 1997)

$$
\dot{M}=\frac{\sqrt{18 \pi^{3}} \alpha_{\text {disk }} V_{c} \rho_{0} h_{0}^{3}}{R_{*}},
$$

where the critical velocity $V_{c}=\left(G M_{*} / R_{*}\right)^{1 / 2}$. The flux due to viscous disk accretion, $G M_{*} M / 2 R_{*}$, is generated throughout the disk midplane region according to

$$
\frac{d E}{d A d t}=\frac{3 G M_{*} \dot{M}}{4 \pi \varpi^{3}}\left(1-\sqrt{\frac{R_{*}}{\varpi}}\right) .
$$

We adopt $M_{*}=0.5 M_{\odot}$ and vary $\alpha_{\text {disk }}$ to place limits on the accretion luminosity for a given $M_{\text {disk }}$.

The variability of $\mathrm{HH} 30$ 's scattered-light nebula (Burrows et al. 1996; Stapelfeldt et al. 1998; Cotera et al. 2001) and ground-based VRI photometry (Wood et al. 2000) indicates nonuniform illumination of the disk caused by either stellar hot spots or shadowing due to large inhomogeneities in the inner disk. D'Alessio et al. (1998), however, showed that hot accretion rings do not significantly alter the SED, so we assume a uniformly bright star and do not include disk warping in our models.

\subsection{Circumstellar Dust Properties}

Burrows et al. (1996) fitted their HH 30 IRS HST images using a dust-scattering phase function that was more forward throwing than ISM dust grains, and the near-IR modeling by Cotera et al. (2001) derived a shallower wavelength-dependent opacity than that of ISM grains. Both these results are indicative of circumstellar dust grains that are larger (in a statistically significant sense, e.g., crosssection weighted mean size) than typical ISM grains. Grain growth within protoplanetary disks has been inferred from millimeter observations and the slope of long-wavelength SEDs - millimeter fluxes are larger and the SED slopes are shallower than predicted from ISM grains (e.g., Beckwith et al. 1990; Beckwith \& Sargent 1991). In order to examine the possibility of grain growth within the HH 30 disk as well as the potential characteristics thereof, we construct models with several different dust size distributions: a standard ISM mixture and several distributions with larger grains including both simple power laws and power laws with an exponential cutoff.

For the ISM dust, we adopt the size (mass) distribution of Kim, Martin, \& Hendry (1994, hereafter KMH) for the canonical diffuse interstellar sight line (i.e., $R_{V}=3.1$ ). In addition, we also reproduce the model of Cotera et al. (2001). The interested reader is referred to these two works for specific details of the models.

For our "larger grain" dust models, we combine a "power-law with exponential cutoff" (e.g., KMH) for components of amorphous carbon and astronomical silicates with solar abundance constraints for carbon and silicon $\left(\mathrm{C} / \mathrm{H} \sim 3.5 \times 10^{-4}\right.$ and $\mathrm{Si} / \mathrm{H} \sim 3.6 \times 10^{-5}$, respectively; Anders \& Grevesse 1989; Grevesse \& Noels 1993). The primary motivation for introducing an additional complexity onto a simple power law (one with a single exponent; e.g., D'Alessio et al. 2001) is that it is not expected to apply over such a large range of grain sizes. This has been indicated in analyses of both ISM and circumstellar dust grains (e.g., KMH; Jones, Tielens, \& Hollenbach 1996; Witt, Smith, \& Dwek 2001; Suttner \& Yorke 2001). In addition, rather than joining two separate power laws (i.e., Witt, Smith, \& Dwek 2001), we adopt a distribution that provides a smooth transition between two different regimes and that can reproduce the general behavior of a large range of interstellar sight lines (e.g., KMH). The functional form of this size distribution is

$$
n(a) d a=C_{i} a^{-p} \exp \left[-\left(\frac{a}{a_{c}}\right)^{q}\right] d a .
$$


TABLE 1

DUST MODEL PARAMETERS

\begin{tabular}{lcccccr}
\hline \hline Dust Model & Component & $C_{i}$ & $p$ & $q$ & $\begin{array}{c}a_{c} \\
(\mu \mathrm{m})\end{array}$ & $\begin{array}{r}a_{\max } \\
(\mu \mathrm{m})\end{array}$ \\
\hline Cotera $\ldots \ldots .$. & Amorphous carbon & $5 \times 10^{-16}$ & 3.5 & 1.0 & 0.55 & 20 \\
& Silicon & $6 \times 10^{-16}$ & 3.0 & 1.0 & 0.55 & 20 \\
Model 1...... & Amorphous carbon & $1.32 \times 10^{-17}$ & 3.0 & 0.6 & 50 & 1000 \\
& Silicon & $1.05 \times 10^{-17}$ & 3.0 & 0.6 & 50 & 1000 \\
Model 2...... & Amorphous carbon & $1.32 \times 10^{-17}$ & 3.5 & $\infty$ & $\ldots$ & 1000 \\
& Silicon & $1.05 \times 10^{-17}$ & 3.5 & $\infty$ & $\ldots$ & 1000 \\
Model 3...... & Amorphous carbon & $1.32 \times 10^{-17}$ & 3.0 & $\infty$ & $\ldots$ & 1000 \\
& Silicon & $1.05 \times 10^{-17}$ & 3.0 & $\infty$ & $\ldots$ & 1000 \\
\hline
\end{tabular}

NoTE.-Size distribution for the KMH model is taken from their mass distribution for the fit to the canonical $\left(R_{V}=3.1\right)$ average diffuse ISM sight line.

The parameters $p, a_{c}$, and $q$, which control the distribution shape, are adjusted to fit the wavelength dependence of the observations. Of course, a simple power law corresponds to the case of $q=\infty$. The term $C_{i}$ is set by requiring the grains to completely deplete a solar abundance of carbon and silicon. The subscript $i$ refers to the different components of the dust model: amorphous carbon and silicate (see Table 1). This function is essentially the "modified gamma distribution" found in atmospheric and planetary science literature. The endpoints of the size integration, $a_{\min }$ and $a_{\max }$, are also parameters. Not surprisingly, models with finite values of $q$ are insensitive to the exact value of $a_{\max }$ as long as it is $\gg a_{c}$.

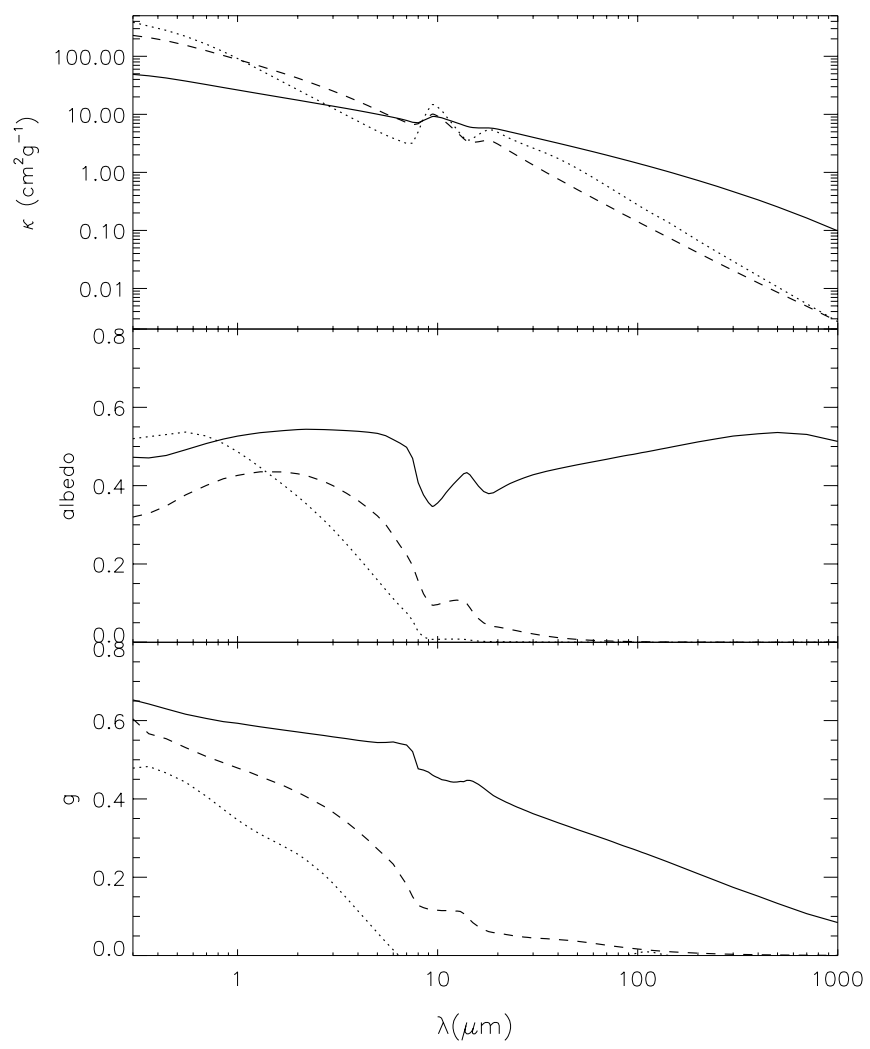

FIG. 2.-Dust parameters for ISM grains (dotted lines), size distribution used by Cotera et al. (2001) (dashed lines), and the size distribution (model 1) we use for fitting the HH 30 SED (solid lines).
The opacity of the dust grains is modeled under the assumption of two separate populations of homogeneous, spherical particles. In general, we use published dielectric functions for astronomical silicate (Weingartner \& Draine 2001) and amorphous carbon (BE type; Zubko et al. 1996). However, our desired wavelength range extends longward of both sets of dielectric functions and slightly shortward of the amorphous carbon values (i.e., 100-500 $\AA$ not in published set). We use a subtractive Kramers-Kronig analysis (cf. Ahrenkiel 1971; routine kindly supplied by K. Snook), specifying the imaginary index of refraction in the regions of interest. This approach also requires specification of the real index of refraction at some reference point; we

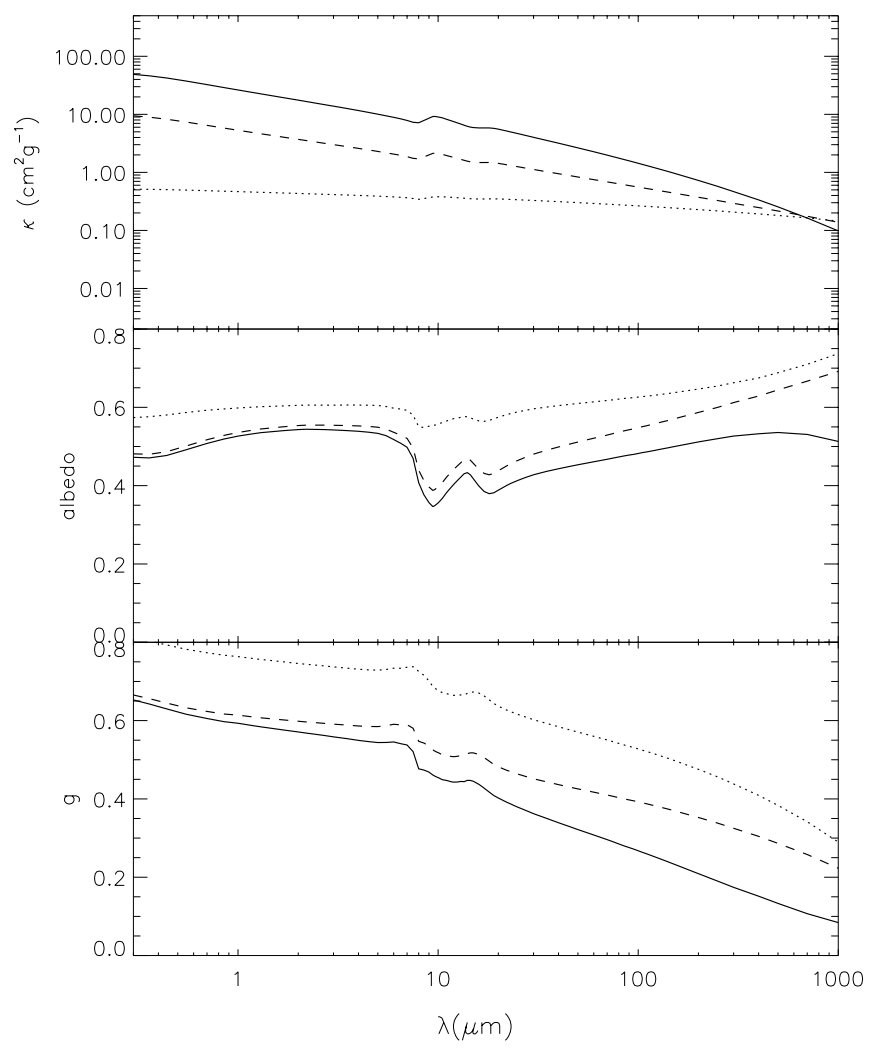

Fig. 3.-Dust parameters for the exponential cutoff dust model (solid line, model 1) and simple power-law models, $p=3.5$ (dashed line, model 2), $p=3.0($ dotted line, model 3). 
use a value of the real refractive index at a wavelength adjacent to the missing range from the published data. For the long-wavelength extension, we use the approximation that imaginary index is proportional to $\lambda^{-N}$ (e.g., Pollack et al. 1994): $N=-1$ for the silicates and $N=\frac{1}{6}$ for the amorphous carbon. The latter choice departs from the recommendation of Pollack et al. for metals $(N=1)$ but is a much better match for the slope of the published data in the far-IR region. Fortunately, our model fits are fairly insensitive to the exact choice of $N$. For the short-wavelength extension, we use the imaginary index of the BE1 of Rouleau \& Martin (1991) scaled to match smoothly with that of Zubko et al. (1996)

The scattering properties are calculated using a combination of Mie series solution and geometrical optics algorithms. For the former, we employ the code of Wiscombe $(1996)^{5}$ while the latter regime is handled with the code of Macke and collaborators (rt-ellipsoid; i.e., Macke \& Mishchenko 1996). ${ }^{6}$ The transition point between the two algorithms is made at a size parameter of 20,000. Though this choice is somewhat arbitrary, at this point the forwardscattering properties as calculated by each algorithm differ at a level below $1 \%$ (the phase matrix elements are in agreement at the $5 \%-10 \%$ level). This is also true at a size parameter of 10,000 , but we chose the larger value in order to allow the phase functions in the visible and near-IR to be calculated entirely by the Mie algorithms; this avoids introducing the small-scale $(\sim 10 \%$ outside the central diffraction peak) high-frequency oscillations produced by the geometrical optics algorithm.

Figures 2 and 3 show the spectral shape of the opacity, albedo, and cosine asymmetry parameter (i.e., $g$ ) for the various dust models we consider. The size distribution parameters are listed in Table 1. Although we use Mie theory for calculating the scattering properties, in the radiative equilibrium calculation we adopt a simple HeyneyGreenstein scattering phase function characterized by the single parameter $g$ (Heyney \& Greenstein 1941).

\section{MODEL SEDs}

A tentative spectral type of M0 for the illuminating source of HH 30 IRS was assigned by Kenyon et al. (1998). Because the central star is totally obscured by the disk at optical and near-IR wavelengths, however, the spectral type is very uncertain. Scattered-light models indicate $L_{*} \approx 0.2$ $L_{\odot}$, so we adopt $T_{*}=3500 \mathrm{~K}$ (Kurucz model atmosphere; Kurucz 1994) and $R_{*}=1.2 R_{\odot}$. The distance to HH 30 IRS is taken to be $140 \mathrm{pc}$. The following sections show the resulting SEDs for different dust models, starting with a purely passive reprocessing disk. For each dust model we adopt the disk size and structure described in $\S 3.2$ and determine the disk mass from fitting the NICMOS F160W image. We then calculate the model SED for comparison with the data presented in Figure 1. Figure 4 shows the NICMOS F160W image and scattered-light model fits for the five dust types described in $\S 3.4$ and summarized in Table 2. The $A_{V}$ values in this table are calculated differently than those presented by Cotera et al. (2001). We have

\footnotetext{
${ }^{5}$ Document and source code available at $\mathrm{ftp}: / /$ climate.gsfc.nasa.gov/ pub /wiscombe/Single_Scatt/Homogen_Sphere/Exact_Mie/.

${ }^{6}$ Source code available at http://www.ifm.uni-kiel.de/me/research/ Projekte/RemSens/SourceCodes/source.html.
}
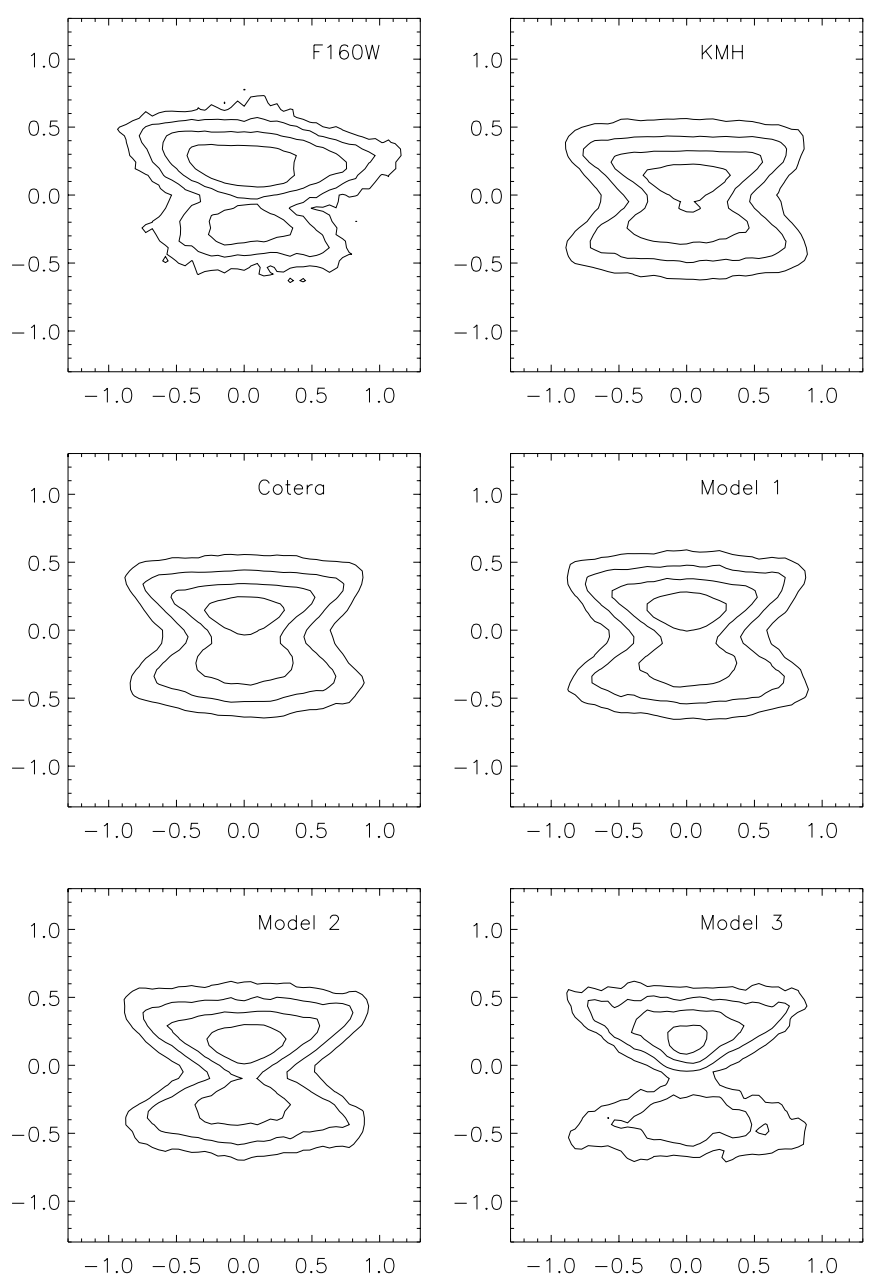

FIG. 4.-NICMOS F160W image of HH 30 IRS and scattered-light models for the different dust size distribution models investigated in this paper. Axes are labeled in arcseconds, where $1^{\prime \prime}=140$ AU.

simply integrated through the disk along a viewing angle of $i=84^{\circ}$, whereas Cotera et al. measured how much light escapes in the Monte Carlo simulation to get an effective $A_{V}$.

The width of the dust lane in the scattered-light images is sensitive not only to the disk mass (and therefore $\tau_{\text {eq }}$ and $A_{H}$ ), but also to the scattering phase function. If $g$ were the same for all models then $A_{H}$ would be the same also. As the phase function becomes more forward throwing for the larger dust grain models (i.e., $g$ increases), however, a larger optical depth is required to obtain the same width of the dust lane.

TABLE 2

DisK MASSES AND EXTINCTIONS

\begin{tabular}{llrrrrr}
\hline \hline $\begin{array}{c}\text { Dust } \\
\text { Model }\end{array}$ & $M_{\text {disk }} / M_{\odot}$ & \multicolumn{1}{c}{$A_{H}$} & $\tau_{\text {eq }}(H)$ & $\mathrm{g}(H)$ & \multicolumn{1}{c}{$A_{V}$} & $A_{1.3 \mathrm{~mm}}$ \\
\hline KMH .... & $3.5 \times 10^{-4}$ & 800 & 31000 & 0.28 & 4600 & 0.05 \\
Cotera .... & $3.5 \times 10^{-4}$ & 1000 & 39000 & 0.49 & 2800 & 0.05 \\
Model 1... & $1.5 \times 10^{-3}$ & 1800 & 65000 & 0.58 & 3300 & 7.9 \\
Model 2... & 0.015 & 3700 & 130000 & 0.60 & 6500 & 120 \\
Model 3... & 0.5 & 13000 & 440000 & 0.75 & 15000 & 4200 \\
\hline
\end{tabular}

NoTE.-Optical depths are in the disk midplane and extinctions are along $i=84^{\circ}$. 


\subsection{Reprocessing Disk SEDs for $i=84^{\circ}$}

Figures 5 and 6 show the HH 30 IRS data and model SEDs (for $i=84^{\circ}$ derived from scattered-light models) for the dust types described in $\S 3.4$ and summarized in Table 1 . The models show the characteristic double-peaked SEDs of highly inclined disks. The disk mass has been chosen so that each model matches the NICMOS F160W image (Cotera et al. 2001). The different dust models are distinguished from one another at longer wavelengths. Although the ISM grains and the Cotera et al. grains produce similar millimeter fluxes, they differ in the $1-10 \mu \mathrm{m}$ range where the ISM grains have a steeper wavelength-dependent opacity that yields too narrow a dust lane in NICMOS scattered-light images. The millimeter fluxes are best reproduced with larger grain-size distributions. Our pure reprocessing disk models yield a rather low flux in the $1-10 \mu \mathrm{m}$ range, and $\S 4.3$ shows models including accretion luminosity that provide a better fit to this spectral regime.

The different models, disk mass estimates, midplane optical depths $\left(\tau_{\text {eq }}\right)$, and extinction to the star for $i=84^{\circ}$ are summarized in Tables 1 and 2. The simple power-law size distributions can have very large millimeter optical depths. While dust size distributions with small $a_{\max }$ can be used to fit individual scattered-light images, the millimeter fluxes are best fit with the large grain models. These models require very different masses and our SED models show that they may be distinguished in the mid- to far-IR mea-

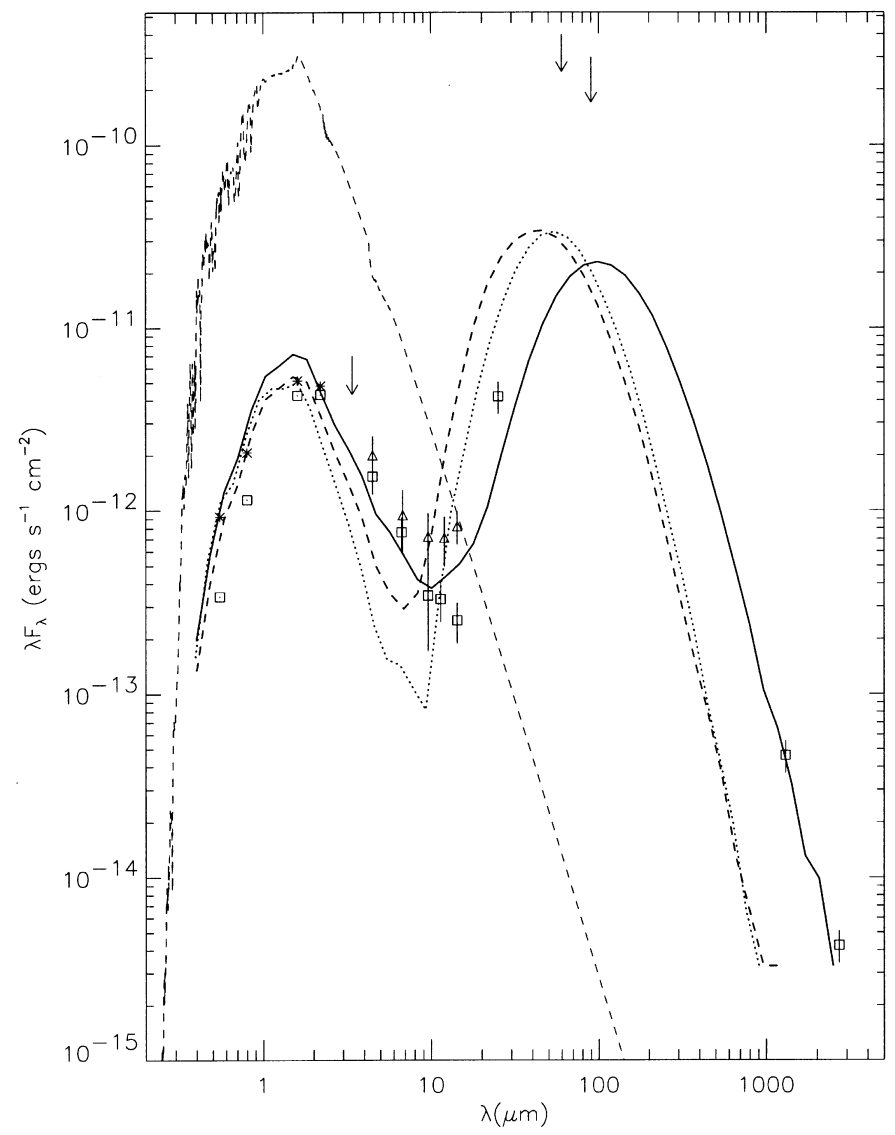

FIG. 5.-Data and model SEDs for passive disks for the three different dust models presented in Fig. 2. Stars show dereddened fluxes assuming $A_{V}=1$. Model SEDs for $i=84^{\circ}$ are for ISM grains (dotted line), Cotera et al. (2001) grains (dashed line), exponential cutoff (solid line, model 1). The input stellar spectrum is shown and the adopted source distance is $140 \mathrm{pc}$.

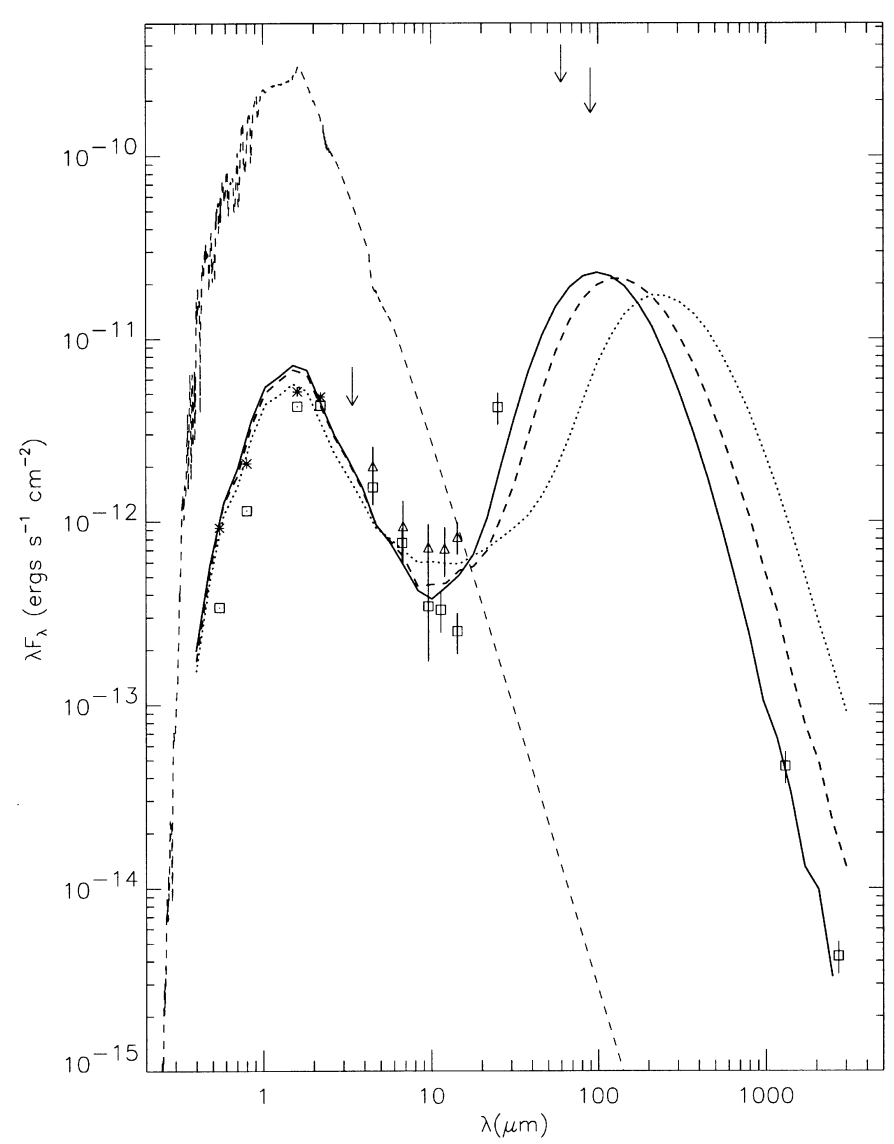

Fig. 6.-Data and model SEDs for the three different dust models presented in Fig. 3. Stars show dereddened fluxes assuming $A_{V}=1$. Model SEDs are for the exponential cutoff (solid line, model 1), and simple power laws, $p=3.5$ (dashed line, model 2), $p=3.0$ (dotted line, model 3). The input stellar spectrum is also shown. The models may be discriminated by the SED in the range $20 \mu \mathrm{m} \lesssim \lambda \lesssim 1 \mathrm{~mm}$.

sured by SIRTF and in the submillimeter measured by SubMillimeter Array (SMA) and Atacama Large Millimeter Array (ALMA). That is to say, although a simple power law is not physically plausible in objects like HH 30 IRS, the issue of whether it adequately reproduces the SED can be determined with sufficient observation coverage.

\subsection{Reprocessing Disk SEDs for All Inclinations}

Scattered-light modeling of HH 30 IRS indicates that we are seeing a classical $\mathrm{T}$ Tauri star viewed almost edge-on. Whitney \& Hartmann (1992) presented scattered-light disk models for a range of viewing angles showing that edge-on disks, where the central star is obscured, will be most easily detectable. Our edge-on SED models agree well with the current HH 30 IRS data, and Figure 7 shows model SEDs at a range of viewing angles. As expected, for viewing angles where the central source is unobscured, our HH 30 IRS models do indeed resemble the SED of a classical T Tauri star. The silicate features at 9 and $18 \mu \mathrm{m}$ are most prominent in the ISM dust model. As the grain size increases and the opacity is dominated by larger grains, the relative strengths of the silicate features decrease (Fig. 2). This is reflected in the SEDs with the $a_{c}=50 \mu \mathrm{m}$ size distribution showing a very smooth SED across the silicate features. At present, however, our models do not allow for different dust compositions at different locations within the disk as 


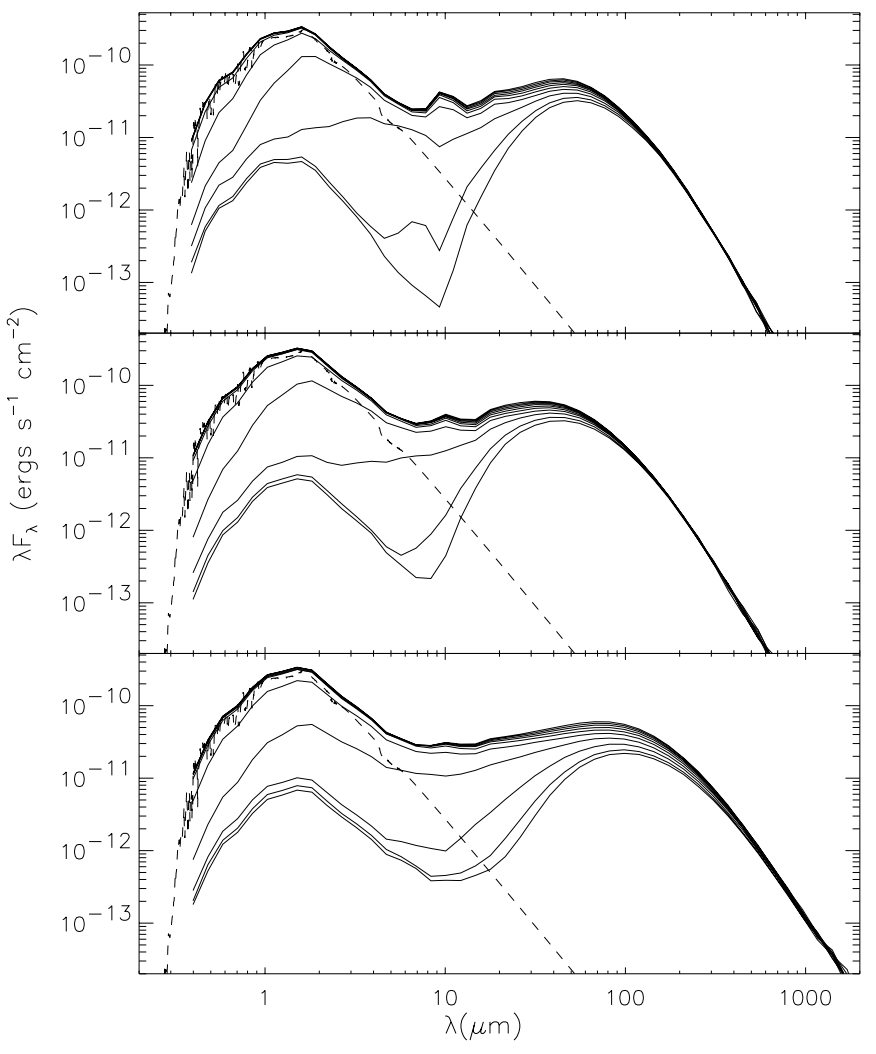

FIG. 7.-Model SEDs at 10 viewing angles evenly spaced in cos $i$ for KMH grains (upper panel), Cotera et al. (2001) (middle panel), and the size distribution we use for fitting the HH 30 IRS SED (lower panel, model 1). Notice the decrease in the strength of the silicate features at 9 and $18 \mu \mathrm{m}$ for the larger grain models.

required for a detailed investigation of the disk chemistry and mineralogy through analysis of crystalline features (e.g., Chiang et al. 2001).

\subsection{Effects of Accretion Luminosity}

HH 30 IRS is the source of a powerful jet (Mundt \& Fried 1983; Mundt et al. 1990; Graham \& Heyer 1990; Lopez et al. 1995), presumably driven by accretion luminosity. The low disk mass for $\mathrm{HH} 30$ IRS derived using ISM dust models is unlikely to support a large accretion rate in the context of $\alpha$-disk theory. Therefore, the larger disk masses we derive are more appealing as they allow for significant accretion rates. Figure 8 shows models that include disk heating from viscous accretion for model 1 , which has $M_{\text {disk }}=1.5 \times 10^{-3} M_{\odot}$.

We find $L_{\text {acc }}=0.2 L_{*}$ provides a good match to the 1-10 $\mu \mathrm{m}$ fluxes presented by Brandner et al. (2000). Assuming $M_{*}=0.5 M_{\odot}$ then $\dot{M}=4 \times 10^{-9} M_{\odot} \mathrm{yr}^{-1}$ and $\alpha_{\text {disk }}=$ 0.02 . A larger accretion luminosity produces too much $1-10$ $\mu \mathrm{m}$ emission compared to the observations. Figure 8 also shows models in which the accretion luminosity is lower $\left(L_{\text {acc }} / L_{*}=5 \%, 2 \%\right)$. Pole-on viewing yields typical near-IR excesses arising from accretion disks.

The large uncertainties in the $1-10 \mu \mathrm{m}$ fluxes in addition to the unknown $M_{*}$ and $\alpha_{\text {disk }}$ preclude an accurate determination of the accretion rate. However, the accretion rate we derive is comparable to the total outflow rate in the jets $\dot{M}_{\text {jet }}=3.5 \times 10^{-9} M_{\odot} \mathrm{yr}^{-1}$ estimated by Bacciotti, Eisloffel, \& Ray (1999). The combination of SED models of more accurate SIRTF fluxes and spectral line accretion diagnos-

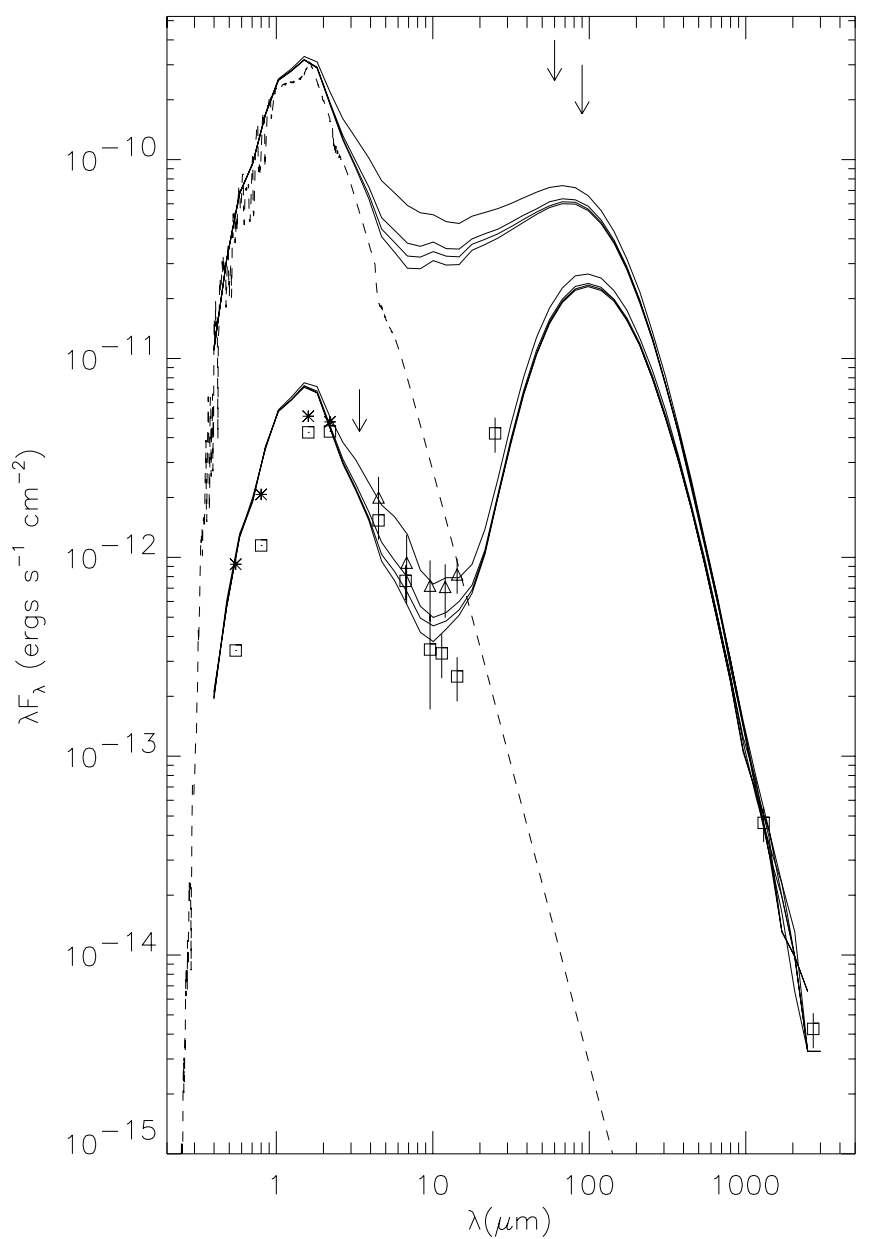

FIG. 8.-Data and model SEDs including accretion luminosity. The model SEDs are for $i=84^{\circ}$ (lower curves) and pole-on models (upper curves). For the different viewing angles going from upper to lower curves the accretion luminosity is $L_{\text {acc }} / L_{*}=0.2,0.05,0.05$, and 0.0 .

tics (e.g., Gullbring et al. 1998) are required for a more accurate determination of the accretion rate.

\subsection{Components of the SED}

A feature of Monte Carlo radiation transfer is that we may track the history of each individual photon packet within the simulation and determine the relative contributions to the total SED of direct, scattered, reprocessed, and accretion luminosity. Figure 9 shows model SEDs for dust model 1 at inclinations $i=0^{\circ}, i=60^{\circ}, i=71^{\circ}$, and $i=84^{\circ}$, along with their component parts. The scattered light comprises stellar photons that have been (multiply) scattered; thermal photons are stellar photons that have been absorbed, reprocessed, and possibly scattered by the disk; accretion photons are those that initiated as accretion luminosity, but may have subsequently been scattered or absorbed and reradiated.

For pole-on viewing, the SED is dominated by direct starlight at optical wavelengths and thermal (i.e., reprocessed) disk emission at long wavelengths. Accretion luminosity contributes significantly at near- and mid-IR wavelengths, while scattered starlight contributes a few percent of the SED in the optical and near-IR and much less at longer wavelengths. Scattering will always yield an excess above the photospheric level (see also Whitney \& Hartmann 1992). For edge-on viewing, the situation is different: 


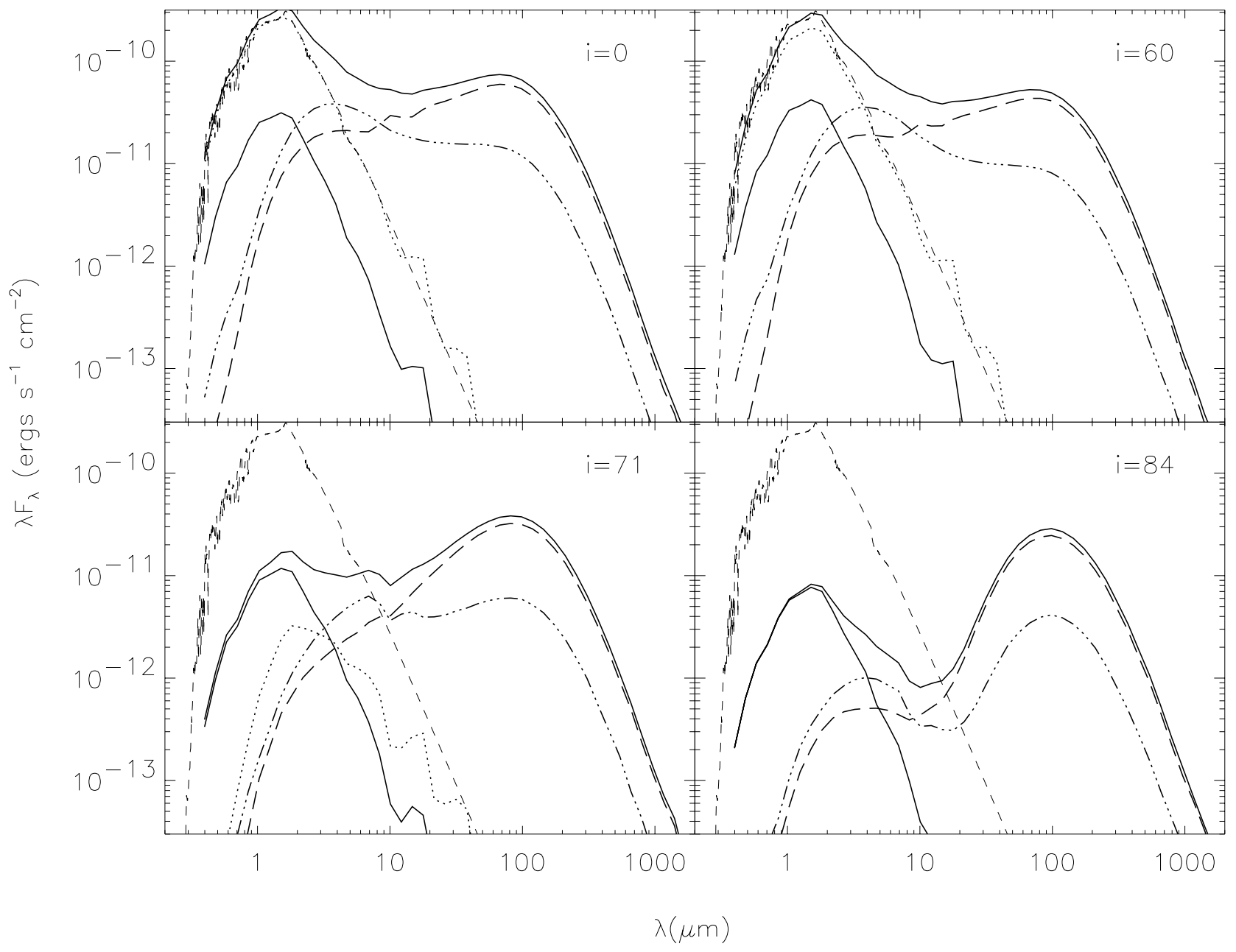

FIG. 9.- Model showing the component parts of the SED for dust model 1 at inclinations $i=0^{\circ}, i=60^{\circ}, i=71^{\circ}$, and $i=84^{\circ}$ (lower panel). Each panel shows the input stellar spectrum (short-dashed line), total SED (uppermost solid line), direct photons (dotted line), scattered starlight (lowest solid line), photons reprocessed by the disk (long-dashed line), and accretion luminosity (dash-triple-dotted line). Direct starlight is not detectable for $i=84^{\circ}$ and scattered starlight dominates the SED at optical and near-IR wavelengths where the star is totally obscured by the flared disk.

the star is totally obscured by the disk and scattered starlight dominates the SED in the optical and near-IR.

\section{SUMMARY}

We have presented Monte Carlo radiation transfer calculations for the SED of HH 30 IRS. Our model comprises a central stellar source surrounded by a circumstellar disk whose structure was derived from modeling HST images. The dust opacity in the disk has a shallower wavelength dependence than typical ISM dust and the average grain size is significantly larger. The model that matches the observed SED has a dust size distribution extending to larger grain sizes than ISM dust; specifically $a_{\max }=1 \mathrm{~mm}$ with an exponential cutoff scale length of $a_{c}=50 \mu \mathrm{m}$. The opacity at long wavelengths is roughly $\kappa \sim \lambda^{-1}$, in agreement with that derived from submillimeter continuum SEDs in many classical T Tauri stars (Beckwith et al. 1990). While size distributions with most of the mass in smaller grains can be constructed to fit optical and near-IR images, these grain models produce too little millimeter emission. The disk mass we derive using the model above is $M_{\text {disk }}=$ $1.5 \times 10^{-3} M_{\odot}$, considerably larger than the $M_{\text {disk }}=2$ $\times 10^{-4} M_{\odot}$ found by Wood et al. (1998) using ISM grains.
The derived disk mass is a lower limit, since the SED is not very sensitive to large particles, such as rocks and planetesimals. Estimations of disk mass from millimeter observations are uncertain due to the poorly known opacity at millimeter wavelengths. For HH 30's disk, Reipurth et al. (1993) estimated $M_{\text {disk }}=0.02 M_{\odot}$ if $\kappa_{1.3 \mathrm{~mm}}=0.003 \mathrm{~cm}^{2}$ $\mathrm{g}^{-1}$ or $M_{\text {disk }}=0.003 M_{\odot}$ if $\kappa_{1.3 \mathrm{~mm}}=0.02 \mathrm{~cm}^{2} \mathrm{~g}^{-1}$. The opacity for our grain size distribution is $\kappa_{1.3 \mathrm{~mm}}=0.08 \mathrm{~cm}^{2}$ $\mathrm{g}^{-1}$, again demonstrating the sensitivity of the disk mass estimate to the adopted millimeter opacity. Observations with SIRTF and at submillimeter wavelengths will give a more complete picture of the SED of HH 30 IRS and other edge-on disks, allowing us to further constrain the dust size distribution and ascertain the extent of the grain growth within the disk. In particular, the SED in the $20-800 \mu \mathrm{m}$ range will discriminate between the dust model with exponential cutoff (corresponding to $M_{\text {disk }}=1.5 \times 10^{-3} M_{\odot}$ ) and the single power-law size distributions that require much more massive disks $\left(M_{\text {disk }} \sim 0.5 M_{\odot}\right)$.

Grain growth in protoplanetary disks has been inferred from observations: e.g., shallower slopes of the long wavelength SEDs and larger millimeter fluxes compared with those expected for ISM dust parameters (Beckwith et al. 
1990; Beckwith \& Sargent 1991); the observed wavelength dependence of dust lane widths in near-IR images of HH 30 IRS (Cotera et al. 2001). Our SED modeling of HH 30 IRS provides further evidence for grain growth and suggests larger grain sizes than those derived from scattered-light models only. Grain growth within protoplanetary disks is expected on timescales of $10^{5} \mathrm{yr}$ (Beckwith, Henning, \& Nakagawa 2000, and references therein). The simulations of Suttner \& Yorke (2001) find that compact grains can grow to $a=50 \mu \mathrm{m}$ within $\sim 10^{4} \mathrm{yr}$. In their model, however, the largest grains are found primarily in the disk midplane regions and as such will not have a big influence on the infrared SED, which is dominated by the upper layers or disk "atmosphere." Although the current implementation of our code adopts the same grain size distribution throughout the disk, our SED modeling is consistent with grain growth to larger than $50 \mu \mathrm{m}$, even in the upper layers of the disk atmosphere that dominate the infrared SED.
The mass that we derive for HH 30's disk is more appealing than the low disk mass derived from ISM dust models that are unlikely to support large accretion rates. More massive disks support larger accretion rates and for $\mathrm{HH} 30$ IRS we infer an accretion luminosity of $L_{\text {acc }} \approx 0.2 L_{*}$. Our derived accretion rate, $\dot{M}=4 \times 10^{-9} M_{\odot} \mathrm{yr}^{-1}$, is uncertain as it depends on the unknown $M_{*}$ and $\alpha_{\text {disk }}$ viscosity parameter, and the accurate determination of $\dot{M}$ requires spectral line observations in addition to SED modeling.

We acknowledge financial support from NASA's Long Term Space Astrophysics Research Program, NAG 5-6039 (K. W.), NAG 5-8412 (B. W.), NAG 5-7993 (M. W.), NAG 5-3248 (J. E. B.); the National Science Foundation, AST 99-09966 (B. W. and K. W.), AST 98-19928 (J. E. B.), and a PPARC Advanced Fellowship (K. W.). We thank Debbie Padgett and Karl Stapelfeldt for providing their ISO and OVRO SED data.
Anders, E., \& Grevesse, N. 1989, Geochim. Cosmochim. Acta, 53, 197

Ahrenkiel, R. K. 1971, Appl. Opt., 61, 1651

Bacciotti, F., Eisloffel, J., \& Ray, T. P. 1999, A\&A, 350, 917

Beckwith, S. V. W., Henning, T., \& Nakagawa, Y. 2000, in Protostars and Planets IV, ed. V. Mannings, A. P. Boss, \& S. R. Russell (Tucson: Univ. Arizona Press), 533

Beckwith, S. V. W., \& Sargent, A. I. 1991, ApJ, 381, 250

Beckwith, S. V. W., Sargent, A. I., Chini, R. S., \& Gusten, R. 1990, AJ, 99, 924

Bjorkman, J. E. 1997, in Stellar Atmospheres: Theory and Observations, ed. J. P. De Greve, R. Blomme, \& H. Hensberge (New York: Springer), 239

Bjorkman, J. E., \& Wood, K. 2001, ApJ, 554, 615

Boss, A. P., \& Yorke, H. W. 1996, ApJ, 469, 366

Brandner, W., et al. 2000, A\&A, 364, L13

Burrows, C. J., et al. 1996, ApJ, 473, 437

Cardelli, J. A., Clayton, G. C., \& Mathis, J. S. 1988, ApJ, 329, L33

Chiang, E. I., Joung, M. K., Creech-Eakman, M. J., Qi, C., Kessler, J. E., Blake, G. A., \& van Dishoeck, E. F. 2001, ApJ, 547, 1077

Cotera, A. S., et al. 2001, ApJ, 556, 958

D'Alessio, P., Calvet, N., \& Hartmann, L. 2001, ApJ, 553, 321

D’Alessio, P., Calvet, N., Hartmann, L., Lizano, S., \& Canto, J. 1999, ApJ, 527,893

D’Alessio, P., Canto, J., Calvet, N., \& Lizano, S. 1998, ApJ, 500, 411

Graham, J. A., \& Heyer, M. H. 1990, PASP, 102, 972

Grevesse, N., \& Noels, A. 1993, in Origin and Evolution of the Elements, ed. N. Prantzos, E. Vangioni-Flam, \& M. Casse (Cambridge: Cambridge Univ. Press), 15

Gullbring, E., Hartmann, L., Briceno, C., \& Calvet, N. 1998, ApJ, 492, 323

Hansen, J. E., \& Travis, L. D. 1974, Space Sci. Rev., 16, 527

Henyey, L. C., \& Greenstein, J. L. 1941, ApJ, 93, 70

Jones, A. P., Tielens, A. G. G. M., \& Hollenbach, D. J. 1996, ApJ, 469, 740

Kenyon, S. J., Brown, D. I., Tout, C. A., \& Berlind, P. 1998, AJ, 115, 2491

Kim, S. H., Martin, P. G., \& Hendry, P. D. 1994, ApJ, 422, 164

Kurucz, R. L. 1994, CD-ROM 19, Solar Model Abundance Model Atmospheres (Cambridge: SAO)

\section{EFERENCES}

Lopez, R., Raga, A. C., Riera, A., Anglada, G., \& Estalella, R. 1995, MNRAS, 274, L19

Macke, A. \& Mishchenko, M. I. 1996, Appl. Opt., 35, 4291

Men'shchikov, A. B., \& Henning, T. 1997, A\&A, 318, 879

Motte, F., \& Andre, P. 2001, A\&A, 365, 440

Mundt, R., \& Fried, J. 1983, ApJ, 274, L83

Mundt, R., Ray, T. P., Buhrke, T., Raga, A. C., \& Solf, J. 1990, A\&A, 232, 37

Pollack, J. B., Hollenbach, D., Beckwith, S., Simonelli, D. P., Roush, T., \& Fong, W. 1994, ApJ, 421, 615

Preibisch, T., Sonnhalter, C., \& Yorke, H. W. 1995, A\&A, 299, 144

Reipurth, B., Chini, R., Krugel, E., Kreysa, E., \& Sievers, A. 1993, A\&A, 273,221

Rouleau, F., \& Martin, P. G. 1991, ApJ, 377, 526

Shakura, N. I., \& Sunyaev, R. A. 1973, A\&A, 24, 337

Stapelfeldt, K. R., Krist, J. E., Menard, F., Bouvier, J., Padgett, D. L., \& Burrows, C. J. 1998, ApJ, 502, L65

Stapelfeldt, K. R., \& Moneti, A. 1999, in The Universe as Seen by ISO, ed. P. Cox \& M. F. Kessler (ESA-SP 427; Noordwijk: ESA), 521

Stapelfeldt, K. \& Padgett, D. 2001, in ASP Conf. Ser., Science with the ATACAMA Large Millimeter Array, ed. A. Wootten (San Francisco: ASP), in press

Suttner, G., \& Yorke, H. W. 2001, ApJ, 551, 461

Vrba, F. J., Rydgren, A. E., \& Zak, D. S. 1985, AJ, 90, 2074

Weingartner, J. C., \& Draine, B. T. 2001, ApJ, 548, 296

Whitney, B. A., \& Hartmann, L. 1992, ApJ, 395, 529

Wiscombe, W. J. 1996, NCAR Technical Note, NCAR/TN-140+ STR

Witt, A. N., Smith, R. K., \& Dwek, E. 2001, ApJ, 550, L201

Wood, K., Kenyon, S. J., Whitney, B. A., \& Turnbull, M. 1998, ApJ, 497, 404

Wood, K., Wolk, S. J., Stanek, K. Z., Leussis, G., Stassun, K., Wolff, M., \& Whitney, B. 2000, ApJ, 542, L21

Zubko V. G., Mennella, V., Colangeli, L., \& Bussoletti, E. 1996, MNRAS, 282,1321 